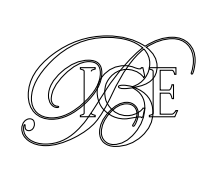

Subdirección General de Fomento y Modernización del Comercio Interior*

\title{
EL PLAN INTEGRAL DE APOYO A LA COMPETITIVIDAD DEL COMERCIO MINORISTA DE ESPAÑA 2017
}

La idea de elaborar un plan que integre las medidas y actuaciones que desarrollan los departamentos ministeriales en el ejercicio de sus competencias y que o bien tienen como objetivo directo o bien puedan favorecer indirectamente al comercio minorista surge en el año 2012, cuando el sector se estaba viendo seriamente afectado por la crisis. En cada edición se ha ido actualizando a las necesidades del momento. Esta iniciativa fortalece la imagen de cohesión y coordinación entre las Administraciones Públicas, a la vez que ofrece de forma completa una descripción de medidas con efectos positivos en potenciar la competitividad del sector del comercio.

Palabras clave: inversión, formación, empleo, competitividad, turismo.

Clasificación JEL: J24, J68, L81, L83.

\section{Introducción}

El Plan de Apoyo a la Competitividad del Comercio Minorista de España 2017 se estructura en diez líneas estratégicas. Desde su primera edición en 2013, con siete ministerios involucrados, se han sumado otros departamentos hasta llegar en 2017 a doce ministerios. El número de medidas también ha ido incrementándose, pasando de treinta a cuarenta y una. En este ejercicio se da mayor preponderancia a la innovación y a la adaptación de los comercios a las nuevas tecnologías y hábitos de consumo que están revolucionando el sector, y la coexistencia de las ventas on y off line es el reto al que se enfrenta el pequeño

\footnotetext{
* Ministerio de Economía, Industria y Competitividad.

Versión de noviembre de 2017.
}

comercio, sin perder de vista el fomento de los centros comerciales abiertos y mercados municipales como formas tradicionales de comercio que mantienen la vida y la actividad en las calles, el apoyo financiero a las empresas para facilitar las iniciativas e inversión, la promoción comercial y la reactivación de la demanda, el fomento de las sinergias entre comercio y turismo potenciando la imagen de nuestro país como destino de compras, el seguimiento de los efectos de la aplicación de las medidas legislativas adoptadas en la eliminación de trabas y obstáculos al ejercicio de la actividad comercial, el impulso a la internacionalización; el apoyo a la seguridad en el comercio y en la protección de las marcas, y las medidas de facilitación del empleo y la formación, que dignifiquen la profesión y aseguren un empleo digno y cualificado. 


\section{El Plan Integral de Apoyo a la Competitividad del Comercio Minorista en 2017}

A continuación se exponen de manera sucinta las principales medidas y novedades que componen cada una de las líneas en que se estructura el plan integral.

\section{Línea 1. Innovación y nuevas tecnologías en el comercio}

Esta línea contiene medidas cuyo objetivo es impulsar la competitividad en el comercio minorista a través de las nuevas tecnologías que están transformando el mundo del comercio como estrategia indispensable de adaptación a los nuevos hábitos de compra y de venta, haciendo de nuestros comercios negocios que sin perder su singularidad sean actuales, modernos e innovadores.

En el marco del convenio de colaboración suscrito por la Secretaría de Estado de Comercio con la Cámara de Comercio de España, las cámaras de comercio llevan a cabo un programa individualizado de innovación consistente en un análisis del establecimiento comercial que proporcione recomendaciones para adoptar metodologías y soluciones que mejoren su situación competitiva y su grado de digitalización, adaptándose a los nuevos desarrollos tecnológicos y a las nuevas formas y hábitos de consumo. Así mismo, se da orientación para la mejora de los establecimientos físicos en la atención a las necesidades en segmentos de población mayores y para la adaptación de comercios con venta on line con un enfoque "age-friendly». En 2017 está previsto realizar 1.316 diagnósticos de innovación.
Las Cámaras también realizan jornadas para mejorar la capacidad de las pymes comerciales en diversas materias estratégicas, que incluyen temáticas como comercio electrónico, marketing digital, turismo de compras, técnicas de venta, nuevos hábitos de consumo y nuevas oportunidades de negocio, entre otras. En 2017 se realizarán 606 talleres con 14.890 asistentes.

Red.es, como entidad pública empresarial responsable del desarrollo de programas de impulso de la economía digital, la innovación, el emprendimiento, la formación y el apoyo a la pyme, desarrolla un programa de fomento de la demanda de soluciones cloud computing para pymes y autónomos y promueve la transformación digital de las pymes mediante asesores digitales y oficinas de transformación digital, así como ayudas directas para la incorporación de soluciones TIC en los negocios.

En esta primera línea estratégica también se incluyen los reconocimientos a la labor en el sector, entre los que se encuentran los Premios Nacionales de Comercio Interior de la Secretaría de Estado de Comercio en sus tres modalidades: Ayuntamientos, Pequeño Comercio y Centros Comerciales Abiertos; el premio a la Producción de la Pesca y la Acuicultura, de MAPAMA; y el sello de calidad para librerías de la Secretaría de Estado de Cultura en colaboración con la Asociación de las Cámaras del Libro de España.

Como novedad, en este ejercicio se está trabajando, desde la Secretaría de Estado de Comercio, la Federación Española de Municipios y Provincias y el Banco Santander, en una convocatoria de un concurso de ideas de soluciones tecnológicas innovadoras para la actividad empresarial del comercio minorista o que incorporen nuevas tecnologías que mejoren la experiencia de compra. 


\section{Línea 2. Modernización de centros comerciales abiertos, mercados municipales y estructuras comerciales}

Esta línea tiene en cuenta el importante papel económico y social de los comercios en las ciudades, ya que dotan de singularidad, atractivo y vida al centro de las ciudades.

Por ello, esta línea aborda medidas orientadas a mejorar los mercados municipales promoviendo su modernización mediante estudios técnicos-comerciales y de viabilidad económi$\mathrm{ca}$, que son realizados por Mercasa, en virtud de una encomienda de gestión de la Secretaría de Estado de Comercio. En 2017 se llevan a cabo estudios de mejora de cinco mercados municipales.

En el marco del convenio de colaboración suscrito entre la Secretaría de Estado de Comercio y la Cámara de Comercio de España, esta última publica una convocatoria abierta de ayudas para la realización de proyectos destinados a la modernización y revitalización comercial de entornos urbanos y la mejora de la competitividad de los equipamientos comerciales, ubicados en zonas de gran afluencia turística y municipios con libertad de apertura. En 2017 se han adjudicado ayudas a 13 proyectos por valor de 1.339.721 euros, que beneficiará a 2.778 comercios y generará una inversión de 2,4 millones de euros.

Además, la Secretaría de Estado de Cultura convoca subvenciones para proyectos de revalorización cultural y modernización de las librerías, dotado con 120.000 euros, y la Dirección General de Relaciones con las Comunidades Autónomas y Entes Locales gestiona 79 Estrategias de Desarrollo Urbano Sostenible e Integrado que incluyen programas de apoyo al comercio minorista en áreas urbanas desfavorecidas o en áreas urbanas de interés turístico.

\section{Línea 3. Apoyo económico y financiero}

En esta línea se incluyen el Fondo de Financiación a Comunidades Autónomas y el Fondo de Financiación a Entidades Locales para garantizar el pago a proveedores, así como las líneas de financiación del Instituto de Crédito Oficial (ICO) a las que pueden tener acceso las empresas del sector del comercio como ICO Empresas y Emprendedores para proyectos de inversión o necesidades de liquidez; ICO Garantía SGR/SAECA ${ }^{1}$, que ofrece financiación para inversiones o necesidades de liquidez de los autónomos y empresas que cuenten con el aval de una SGR o de SAECA; ICO Crédito Comercial, que facilita liquidez a las empresas mediante el anticipo del importe de las facturas procedentes de su actividad comercial así como la liquidez necesaria para la etapa de fabricación de bienes o servicios que vayan a ser objeto de venta; ICO Internacional, que pone a disposición de los autónomos, empresas, emprendedores y entidades públicas y privadas financiación para acometer inversiones fuera del territorio nacional y cubrir las necesidades de liquidez derivadas de su salida al exterior; ICO Exportadores, que facilita las exportaciones mediante el anticipo del importe de sus facturas correspondientes a ventas a corto plazo y la financiación de la etapa de fabricación de bienes o servicios que van a ser objeto de exportación; e ICO-IDAE Eficiencia Energética, destinada a la financiación de actuaciones de mejora de la eficiencia energética en la que el Instituto para la Diversificación y Ahorro de la Energía (IDEA) otorga avales con cargo al Fondo Nacional de Eficiencia para cubrir el 50 por 100 del riesgo de los préstamos concedidos con fondos del ICO.

SGR: sociedad de garantía recíproca. SAECA: Sociedad Anónima Estatal de Caución Agraria. 
Por su parte, el Ministerio de Fomento gestiona ayudas para la rehabilitación edificatoria y la regeneración y renovación urbana que tiene repercusión en los establecimientos comerciales ubicados en los edificios y calles, así como las ayudas para la Rehabilitación Energética de Edificios (PAREER-II), para la reforma energética de todo tipo de edificios con el objetivo de reducir las emisiones de $\mathrm{CO}_{2}$ y del consumo de energía final.

A través de la Compañía Española de Reafianzamiento, SME, SA (CERSA), dependiente de la Dirección General de Industria y de la Pequeña y Mediana Empresa, se ofrece el reafianzamiento o cobertura parcial del riesgo asumido por las Sociedades de Garantía Recíproca (SGR) con las pymes que precisan de garantías adicionales para resolver su problemática financiera, priorizando la financiación de inversiones y proyectos innovadores, así como las microempresas y las de reciente creación.

Además, el Instituto de la Mujer y para la Igualdad de Oportunidades concede una subvención nominativa a CERSA de 75.000 euros para el desarrollo de un Programa de apoyo a la financiación de las mujeres emprendedoras, en el que se subvencionan los gastos de la comisión del estudio realizado por las SGR de los proyectos empresariales de las mujeres emprendedoras.

A ello se añaden las líneas de financiación dirigidas a jóvenes emprendedores que hayan iniciado un negocio de la Empresa Nacional de Innovación ENISA en las primeras fases sin límite de edad o a proyectos empresariales ya conformados enfocados a su expansión y su mejora competitiva.

\section{Línea 4. Promoción comercial}

Sigue siendo de gran trascendencia la fidelización de clientes en la estrategia comercial, por ello se apoya la dinamización de las áreas comerciales y el impulso de las ventas en los establecimientos.

En el marco del convenio de colaboración entre la Secretaría de Estado de Comercio y la Cámara de Comercio de España se lleva a cabo un Programa de Dinamización de Centros Comerciales Urbanos dirigido a incentivar el consumo y las ventas, la fidelización de clientes y la visibilidad de la oferta. Estas actuaciones se llevan a cabo en colaboración con las asociaciones y en coordinación con las comunidades autónomas y los ayuntamientos. En 2017 están previstas 107 campañas que beneficien a 24.320 comercios.

Por su parte, la Secretaría General de Agricultura y Alimentación del Ministerio de Agricultura y Pesca, Alimentación y Medio Ambiente lleva a cabo acciones promocionales destinadas a dar a conocer los beneficios del consumo de los productos que componen la dieta mediterránea, a través de jornadas, carreras populares, talleres, sorteos, concursos y redes sociales, entre otras acciones.

Por último, en esta línea se incluyen programas de promoción sectoriales, como es el caso del Plan MOVEA y PROMOVEA de ayudas para la adquisición de vehículos eléctricos, de gas licuado del petróleo, de gas natural comprimido y licuado y motocicletas eléctricas, así como la implantación de puntos de recarga para vehículos eléctricos en zonas de acceso público. El programa va dirigido tanto a particulares como a la renovación de flotas por parte de las empresas.

\section{Línea 5. Apoyo al emprendedor}

La creación de empresas tiene una gran importancia para la generación de empleo y el crecimiento económico. Por ello, esta línea $\triangleright$ 
incluye el programa de ayudas para el apoyo al emprendimiento en TIC y economía digital de Red.es, que ofrece financiación a empresas de reciente creación que dispongan de una plataforma o tecnología propias.

El Instituto de la Mujer y para la Igualdad de Oportunidades, en colaboración con la Cámara de Comercio de España, desarrolla el Programa de Apoyo al Emprendimiento Femenino (PAEM), que ofrece una atención directa y personalizada, tanto por presencia física como on line, con información sobre oportunidades de autoempleo, legislación, trámites, direcciones de interés, así como asesoramiento especializado en gestión empresarial, planes de viabilidad, análisis de comercio interior y exterior, nuevas tecnologías. También incluye un servicio específico de apoyo a la financiación a través de microcréditos de hasta 25.000 euros sin avales.

A ello se añade el programa Desafío Mujer Rural, destinado a fomentar la creación de pymes por mujeres que viven en el ámbito rural y pesquero, apoyando a las emprendedoras en las distintas fases de su proyecto. Este programa comprende un estudio sobre el emprendimiento rural y la situación y perfil de las emprendedoras; un concurso «Desafío Mujer Rural» para fomentar el autoempleo y la creación de nuevas empresas; el portal de emprendimiento de la mujer rural con una plataforma de comercio electrónico que permite a las emprendedoras vender sus productos y servicios en el ámbito local, nacional e internacional; programas de formación y mentorización en diferentes ámbitos como la innovación, los modelos de negocio, la digitalización, los fundamentos legales y financieros, la gestión comercial, etcétera, o metodologías específicas de lanzamiento de negocios como Lean Start Up y un programa de coaching para acompañar y entrenar a mujeres del medio rural a mejorar sus capacidades que les permita desarrollarse profesionalmente.

La Oficina Española de Patentes y Marcas (OEPM) lleva a cabo distintas actividades de información en ferias donde se informa a los emprendedores sobre las diferentes modalidades de propiedad industrial que se tramitan en la OEPM y los servicios de atención al público. Un ejemplo de ello son las ferias de diseño, de calzado y moda (Futurmoda), del juguete y del sector de alimentación. También difunde libros y ponencias con casos de éxito de empresas españolas.

Por otra parte, la Dirección General de Industrias Culturales y del Libro gestiona la campaña informativa «Cultura en positivo», con un sello que pueden utilizar las empresas e instituciones que se adhieran, y que permite a los usuarios de internet conocer que las páginas web de esas empresas e instituciones ofrecen contenidos culturales digitales en condiciones de legalidad, calidad y seguridad. Asimismo, dispone de un Centro de Documentación en materia de propiedad intelectual con servicio de préstamo de bibliografía y un servicio de información a través de la web del Ministerio de Educación, Cultura y Deporte.

El proyecto CEVIPYME fue creado a iniciativa de la Dirección General de Industria y de la Pequeña y Mediana Empresa, la OEPM y la Cámara de Comercio de España como centro de apoyo a la pyme en materia de gestión de propiedad industrial, intelectual e innovación. Pone a disposición del emprendedor una página web gratuita con información y asistencia personalizada sobre las mejores opciones para la protección de la propiedad industrial, intelectual e innovación, así como información referente a financiación, ayudas o puesta en marcha de ideas de negocio. 
El Consejo para la Unidad de Mercado es un órgano de cooperación administrativa para el seguimiento de la aplicación de la Ley 20/2013, de 9 de noviembre, de Garantía de la Unidad de Mercado (LGUM), en el que están representados los Ministerios de la Presidencia y de Economía, Industria y Competitividad, las comunidades autónomas y ciudades autónomas y las entidades locales. Aprobó la Guía y el Código de buenas y malas prácticas para la aplicación de la LGUM, cuyo objetivo es facilitar la aplicación de la normativa, proporcionar seguridad jurídica y facilitar la identificación de los obstáculos y barreras de la actividad económica que puedan ser contrarios a la LGUM.

\section{Línea 6. Comercio y turismo}

La relación y sinergias entre el comercio y el turismo tienen gran trascendencia para ambos sectores, y no hay que olvidar que en 2016 el turismo en España volvió a batir un récord histórico con la llegada de más de 75,6 millones de turistas extranjeros, lo que supuso un aumento del 10,3 por 100 sobre 2015. En los nueve primeros meses de 2017, España ha recibido la visita de más de 66 millones de turistas internacionales, un 10,1 por 100 más que en el mismo periodo de 2016. En relación con el gasto total realizado por los no residentes, en 2016 ascendió a 77.625 millones de euros, un 9,0 por 100 más que en el mismo periodo de 2015.

Por ello se sigue trabajando en colaboración con Turespaña en la potenciación de España como destino de turismo de compras. En 2017 se realizan 227 actividades para la promoción del mismo, entre las que se encuentran: acciones con medios de comunicación en los mercados emisores de turistas, presentaciones al sector profesional de viajes, viajes de agentes especializados para mostrar la oferta, jornadas directas o inversas centradas en el producto compras y/o lujo en China, India o México, participación en ferias con un componente o presencia destacada de la oferta de turismo de compras (São Paulo, Shanghai, Tokio o París).

En esta línea también se encuentra incluida la agilización de la concesión de visados turísticos principalmente en los mercados emergentes como Rusia, Ucrania, China, Turquía e India por el Ministerio de Asuntos Exteriores y de Cooperación.

\section{Línea 7. Seguridad}

La línea 7 recoge medidas dirigidas a mejorar las condiciones de seguridad de los establecimientos comerciales y de los productos, propiciando una experiencia de compra gratificante, fortalecidas por la proximidad y la comunicación permanente entre los comerciantes y los cuerpos y fuerzas de seguridad del Estado.

La Dirección General de la Policía Nacional difunde consejos de seguridad preventivos mediante una guía práctica de seguridad y un tríptico de comercio seguro con la colaboración de la Dirección General de Política Comercial y Competitividad, de los Delegados de Participación Ciudadana de la Policía Nacional y de las asociaciones de comerciantes, con una nueva edición actualizada en este ejercicio.

Las Delegaciones de Participación Ciudadana y las Brigadas y áreas de Seguridad Ciudadana de la Policía Nacional realizan acciones preventivas con la finalidad de proporcionar un entorno más seguro a los comerciantes. Para ello difunden un cartel disuasorio preventivo con el logotipo de la Policía $\square$ 
Nacional y la indicación «Todos los hurtos y robos detectados en este establecimiento serán denunciados».

La Guardia Civil, por su parte, desarrolla un plan para la mejora de la seguridad en el comercio con planes específicos por tipos de establecimientos y/o zonas geográficas, para dar una respuesta más adecuada a las problemáticas concretas. Trabajan en coordinación con los responsables de seguridad privada implicados en la vigilancia y control de establecimientos comerciales y empresas suministradoras a través de los programas Coopera; así, los establecimientos implicados dispondrán de un cartel con el texto «El personal de seguridad privada de este establecimiento goza de la protección jurídica de agente de la autoridad en los términos del artículo 31 de la Ley 4/2014, de 4 de abril, de Seguridad Privada». Además, se difunde el plan entre la sociedad y el sector con material divulgativo (folletos, carteles y vídeos informativos). Siguen trabajando en la puesta en marcha del proyecto para la implementación de la denuncia electrónica en la Guardia Civil y en la posibilidad de presentación de denuncias in situ que evite el desplazamiento del denunciante al acuartelamiento, procedimientos de actuación conjunta con Cuerpos de Policía Local de aquellas localidades donde se concentran grandes superficies comerciales o zonas urbanas de gran concentración de comercios, con el fin de reducir en lo posible la comisión de hechos delictivos.

Por otra parte, la Oficina Española de Patentes y Marcas impulsa y apoya el desarrollo tecnológico y económico otorgando protección jurídica a las distintas modalidades de propiedad industrial y difunde información relativa a las diferentes formas de protección de la propiedad industrial y contra las falsificaciones a través de la página web de la OEPM (stop-falsificaciones.es), y de la difusión de la guía ¿Qué es una falsificación? en colaboración con la Asociación Nacional para la Defensa de la Marca (ANDEMA). Además, se celebra el día mundial Antifalsificación para concienciar de la importancia de la propiedad industrial y los graves riesgos y perjuicios que conlleva el fenómeno de las falsificaciones, mediante una jornada lúdica, abierta al público, denominada «Marcathlon».

Por otra parte, la Oficina de Recuperación y Gestión de Activos (ORGA) es un órgano dependiente del Ministerio de Justicia al que corresponde localizar y administrar los activos de origen delictivo. Todo ello para que el patrimonio ilícito obtenido como consecuencia del delito no quede en manos del condenado y revierta en beneficio de la sociedad, destinándose una parte a financiar proyectos de apoyo a víctimas y de lucha contra la criminalidad organizada.

La Secretaría de Estado de Comercio colabora con la ORGA facilitando la difusión de su labor y, en concreto, de las subastas de bienes embargados o decomisados en todo el sector comercial. Esta colaboración permite, por una parte, dar mayor difusión a estas subastas, lo que facilitará la venta de los bienes y, por otra parte, supone una oportunidad para el sector comercial que puede estar interesado en adquirir los bienes que se subastan en condiciones económicamente interesantes.

\section{Línea 8. Medidas legislativas de impulso de la actividad comercial y de eliminación de barreras}

El comercio minorista es un ámbito especialmente dinamizador de la actividad económica y del empleo; por ello, en los últimos años, $\triangleright$ 
la legislación ha estado orientada a la reducción de las cargas administrativas y trabas que dificultaban la iniciativa empresarial, permitiendo un régimen más flexible de aperturas, con el objetivo de dinamizar el sector, potenciando la competencia y la estructura productiva de la distribución minorista en España. En estos momentos se hace un seguimiento y evaluación de los efectos de las medidas adoptadas.

Seguimiento de la Ley 12/2012, de 26 de diciembre, de medidas urgentes de liberalización del comercio y de determinados servicios, por la que las licencias municipales de apertura, instalación, funcionamiento, actividad, obras de acondicionamiento y otras se sustituyen por declaraciones responsables o comunicaciones previas en establecimientos de hasta $750 \mathrm{~m}^{2}$. Desde la Dirección General de Política Comercial y Competitividad se hace un seguimiento, en base a los datos aportados por la Agencia Estatal de la Administración Tributaria (AEAT), de los saldos de altas y bajas de los 116 epígrafes de actividades comerciales y de servicios incluidos en el ámbito de aplicación de la ley.

Seguimiento de las declaraciones de Zonas de Gran Afluencia Turística (ZGAT) que se producen de forma obligatoria en base a los criterios del Decreto-ley 20/2012, de 13 de julio, de medidas para garantizar la estabilidad presupuestaria y de fomento de la competitividad relativos a número de habitantes, ocupación hotelera o número de pasajeros en cruceros turísticos, así como de las declaraciones voluntarias de otros municipios. La mayoría de las ZGAT se hallan en comunidades turísticas como son Canarias, Islas Baleares, Cataluña, Valencia y la Comunidad de Madrid.

Actualmente, en toda España, están declaradas 701 zonas de gran afluencia turística en un total de 551 municipios.

\section{Línea 9. Internacionalización}

Teniendo en cuenta que en los últimos años la contribución de la demanda externa al crecimiento ha sido positiva gracias al favorable incremento de las exportaciones, se continúa impulsando, a través del ICEX, la internacionalización de las empresas españolas para contribuir a su competitividad.

EI ICEX cuenta con una amplia batería de medidas. Una de ellas es el programa ICEX next que proporciona asesoramiento especializado y apoyo económico para gastos de prospección, promoción exterior y contratación de personal para el departamento internacional; también apoya la participación en las principales ferias internacionales tanto en los pabellones oficiales que organiza ICEX como en las participaciones agrupadas, coordinadas por las asociaciones.

Desde 2016 el Plan Sectorial de Moda Digital de ICEX y Adigital impulsan la internacionalización de la industria y de las marcas de la moda a través de la transformación digital de la empresa, aplicando innovación e incorporando tecnología en el canal online y en la tienda física.

La Ventana Global es un servicio de información y asesoramiento integral de consultas sobre internacionalización que proporciona desde una plataforma común los servicios y programas de apoyo que ofrecen todos los organismos de la Administración General del Estado: ICEX, ICO, COFIDES, CESCE, CDTI, ENISA; Expansión Exterior y los instrumentos específicos de la Secretaría de Estado de Comercio.

A ello se añaden los servicios personalizados por las cerca de cien Oficinas Económicas y Comerciales repartidas por todo el mundo, que realizan un servicio de consultoría $\triangleright$ 
personalizada y adaptada a las necesidades y peculiaridades del producto.

Por último, a través del programa eMarket Services, se proporciona información y asesoramiento sobre cómo utilizar los mercados electrónicos B2B y B2C para vender en el exterior.

\section{Línea 10. Formación y empleo}

Es indudable la importancia de este sector en el empleo y las necesidades en formación que reclaman. En el año 2016, la ocupación en el comercio minorista creció un 1,7 por 100 en relación al mismo periodo del año anterior. La ocupación creció en el año 2016 en todas las comunidades autónomas más Ceuta y Melilla. Conforme a los datos de la encuesta de población activa el comercio minorista da empleo a más de 1,9 millones, según datos del tercer trimestre de 2017.

Forma parte de esta línea el Sistema Nacional de Garantía Juvenil del Ministerio de Empleo y Seguridad Social, que tiene por finalidad principal que el colectivo de personas jóvenes no ocupadas ni integradas en los sistemas de educación o formación pueda recibir una oferta de empleo, educación continua, formación de aprendiz o periodo de prácticas tras acabar la educación formal o quedar en situación de desempleo.

En este ámbito, el Ministerio de Presidencia y para las Administraciones Territoriales gestiona una línea de subvenciones dirigida a ayuntamientos, diputaciones y similares $u$ organismos autónomos dependientes de ellas, para financiar la realización de actividades formativas para jóvenes, que incluye actuaciones relacionadas con el comercio.

Por su parte, Red.es ha puesto en marcha un Programa de formación para el empleo juvenil en la economía digital con el fin de impulsar la formación y la empleabilidad de los jóvenes desempleados en el ámbito de las TIC y de la economía digital, favoreciendo su capacitación e incentivando su acceso a puestos de trabajo que promuevan la transformación digital de las empresas. Así como ayudas a entidades educativas y universidades para becas a estudiantes de programas formativos de postgrado en el ámbito de la economía digital: comercio electrónico, marketing digital y comunicación online, videojuegos, animación y efectos especiales, programación en tecnologías web y móviles, cloud computing, big data, internet de las cosas (IOT), industria 4.0, smart cities, usabilidad y experiencia de usuario, metodologías de innovación aplicadas a la transformación digital y el emprendimiento, ciberseguridad y realidad virtual y aumentada.

También desarrollan programas formativos para ocupados y para el emprendimiento en pos de la formación continua y reciclaje profesional en temáticas relacionadas con las TIC y la economía digital para favorecer el desarrollo profesional de los trabajadores en la adquisición de competencias digitales e impulsar el proceso de digital de las empresas. Para ello se realizan talleres, jornadas y sesiones de gran capilaridad.

Destaca también la presencia de proyectos sectoriales como el proyecto E-FishNet, que tiene como objetivo promover una red de colaboración internacional sobre los intereses y la mejora formativa en el sector detallista de pescado en Europa, en el que participa un consorcio de nueve organizaciones provenientes de Islandia, España, Turquía, Reino Unido y Portugal. Está enmarcado en el Programa Erasmus +, cofinanciado por la Unión Europea y coordinado por la Federación Española de Pescaderías Tradicionales (FEDEPESCA). $\quad \triangleright$ 
El Observatorio de las Ocupaciones, como unidad técnica del Servicio Público de Empleo Estatal (SEPE), tiene entre sus competencias analizar la situación, las tendencias del mercado de trabajo y los retos y requerimientos que éste plantea, proporcionando un diagnóstico de las necesidades formativas y de recualificación del mercado laboral para anticiparse a los cambios y responder a la demanda que se pueda producir de mano de obra cualificada, contribuyendo así al desarrollo profesional y personal de los trabajadores y a la competitividad de las empresas. En este sentido, en 2017 realiza un estudio prospectivo del sector del comercio minorista en colaboración con la Dirección General de Política Comercial y Competitividad. El objetivo de este estudio es conocer el sector y sus previsiones de futuro, el grado de implantación de las innovaciones más importantes, así como sus carencias formativas y las necesidades de empleo en cuanto a las ocupaciones y perfiles profesionales más relevantes, con la finalidad de adecuar la oferta formativa a las mismas.

La Dirección General de Formación Profesional del Ministerio de Educación, Cultura y Deporte potencia la colaboración de las empresas del sector en el módulo profesional de Formación en Centros de Trabajo (FCT) en los diferentes títulos de formación profesional del sistema educativo, promueve la aplicación de la modalidad de Formación Profesional dual en las empresas del sector del comercio y fomenta la contratación de los titulados de los ciclos formativos de comercio por parte de las empresas con el fin de aumentar la productividad de las propias empresas y poner en valor los títulos de formación profesional en el sector.

Como novedad en este ejercicio, la Dirección General de Política Comercial y
Competitividad, a través de un convenio de colaboración con la Escuela de Organización Industrial (EOI) y en colaboración con la Confederación Española de Comercio (CEC), desarrolla por primera vez acciones de formación en Experiencia de Cliente (Customer Experiencie) para comercios, comenzando con dos proyectos piloto, en 2017, en Albacete y Almería. Su objetivo es concienciar y mentorizar a las pymes de comercio sobre la necesidad de adaptarse a los cambios de comportamiento de los clientes, incorporando el plano emocional en las relaciones con estos y manteniendo un contacto continuo con sus necesidades, hábitos y motivaciones.

\section{Conclusiones}

El plan integral se concibe como un trabajo de colaboración y coordinación de las Administraciones Públicas que engloba las actuaciones que, dentro de las competencias de cada uno de los organismos participantes, se desarrollan con el objetivo de impulsar la competitividad de las pymes del sector del comercio. Estas actuaciones se orientan en distintas líneas, dándose una mayor preponderancia a la innovación y a la adaptación de los comercios a las nuevas tecnologías y a hábitos de consumo que están revolucionando el sector. La coexistencia de las ventas on y off line es el reto al que se enfrenta el pequeño comercio, sin perder de vista la promoción de los establecimientos a pie de calle, la necesidad de financiación, el aprovechamiento de las sinergias con el turismo, potenciando la imagen de nuestro país como destino de compras, la seguridad, la internacionalización y la formación y el empleo. 